\title{
Patient Attitudes towards Surgically Implantable, Long-Term Delivery of Psychiatric Medicine
}

\author{
Farzin Irani ${ }^{1,2}$, Mary Dankert ${ }^{1,2}$, Colleen Brensinger ${ }^{3}$, Warren B Bilker ${ }^{3}$, Sudha R Nair ${ }^{4}$, Christian G Kohler ${ }^{2}$, \\ Stephen J Kanes², Bruce I Turetsky², Paul J Moberg ${ }^{2}$, John D Ragland ${ }^{2}$, Ruben C Gur ${ }^{2}$, Raquel E Gur ${ }^{2}$ and \\ Steven J Siegel*, I,2 \\ 'Division of Neuropsychiatry, Department of Psychiatry, Stanley Center for Experimental Therapeutics, University of Pennsylvania, Philadelphia, \\ PA, USA; ${ }^{2}$ Division of Neuropsychiatry, Department of Psychiatry, University of Pennsylvania, Philadelphia, PA, USA; ${ }^{3}$ Department of Biostatistics \\ and Epidemiology, University of Pennsylvania, Philadelphia, PA, USA; ${ }^{4}$ Horizon House, Community Mental Health Center, USA
}

\begin{abstract}
The introduction of surgically implantable medication delivery systems provides psychiatric patients with reversible, uninterrupted access to medication for up to 14 months. This study designed and administered a survey to assess patients' attitudes and beliefs towards illness, medication, and this potential new treatment method. The survey included questions about demographics, insight and attitudes towards illness, current and past medication adherence, attitudes towards psychiatric and nonpsychiatric medications, and understanding and attitudes towards surgical implants. The sample of 206 psychiatric patients was almost equally split between favorably and unfavorably considering implants. Patients favorable towards implants ascribed forgetting and failure to refill medication on time as the reasons for missing doses, recognized the benefits of medication in general, and understood that the implant would be inserted under the skin. Favorable consideration of implants was positively correlated with the desire to avoid adverse consequences of missing medicine, stay well, avoid the need for daily oral medications, and decrease family burden. Unfavorable consideration of implants was related to a preference to take medication orally, concern about feeling controlled, unwillingness to try something new, and not understanding that the implant would be placed under the skin. Demographic variables, past/current medications, specific diagnosis, and illness severity did not influence the decision. This survey elucidates patients' attitudes and beliefs towards illness, medication, and surgical implants. The results indicate that a significant proportion of patients recognize the difficulties of medication adherence and the need for better methods to attain therapeutic response. Thus, the study provides impetus for future work in this area.

Neuropsychopharmacology (2004) 29, 960-968, advance online publication, I 8 February 2004; doi: I 0.1038/sj.npp. I 300385
\end{abstract}

Keywords: antipsychotic agents; treatment adherence; schizophrenia; drug delivery systems; drug implants; patient compliance

\section{INTRODUCTION}

Medication adherence is a major concern during pharmacological treatment of individuals with serious psychiatric illnesses such as schizophrenia. The acceptance or rejection of a prescribed pharmacological regimen often dictates treatment outcome. Medication nonadherence has been found to result in deterioration of social function, relapse, rehospitalization, poor outcome, and high economic costs (Casper and Regan, 1993; Weiden and Olfson, 1995; Viguera et al, 1997; Bergen et al, 1998). As such, the National

\footnotetext{
*Correspondence: SJ Siegel, Division of Neuropsychiatry, Stanley Center for Experimental Therapeutics in Psychiatry, Clinical Research Building Rm 145a, 4I5 Curie Boulevard, University of Pennsylvania, Philadelphia, PA 19104, USA, Tel: + I 215573 0278, Fax: + I 215 662 7903, E-mail: siegels@mail.med.upenn.edu

Received 04 September 2003; revised 13 December 2003; accepted 16 December 2003

Online publication: 18 December 2003 at http://www.acnp.org/ citations/Npp | 2 1 80303405/default.pdf
}

Institutes of Mental Health has called for the design and evaluation of interventions to help with adherence behaviors (NIMH, 2000). To address this need, we have developed a surgically implantable medication delivery system that would permit psychoactive medication to be delivered for up to 14 months (Siegel et al, 2002; Kahn et al, in review). The current study reports the development and application of a survey that was designed to assess receptiveness towards this new method of medication administration.

Estimates of medication adherence in psychotic patients vary from 7 to $90 \%$ due to variations in definition of compliance, type of population studied, and methods used to assess compliance (Adams and Howe, 1993; Cramer and Rosenheck, 1998; Valenstein et al, 2001). Although several factors have been implicated, it is unclear which models best predict medication adherence. Factors that have been suggested include patient demographics such as age and gender, beliefs and attitudes, insight, patient-provider interaction, medication side effects, attitudes of family 
members as well as quality and severity of symptoms (Francis et al, 1969; Becker and Maiman, 1975; Hogan et al, 1983; David, 1990; David et al, 1992; Awad, 1993; Ghaemi, 1994; Smith et al, 1997; Adams and Scott, 2000). Additionally, the logistics of taking a pill everyday can interfere with medication adherence.

Recently, there has been increased interest in promoting improved medication adherence through new delivery systems across a broad array of medical specialties and diseases. The introduction of transdermal contraceptive patches has allowed better adherence through the systemic delivery of hormones to prevent pregnancy (Archer et al, 2002). Similarly, there have been advances in patch technology to yield novel transdermal therapeutic systems for the delivery of buprenorphine for pain relief (Budd, 2003). Biodegradable polymer microspheres have also been introduced to aid in immunization compliance, which is particularly helpful for developing countries (Gupta et al, 1998). These new methods of medication administration have provided patients with additional options to aid in medication adherence. In psychiatry, current adherence measures include extended release delivery systems lasting from 1 day to 1 week, transdermal patches for nicotine, and long-acting depot antipsychotics (Wei et al, 1996; Fant et al, 1999; Hackett, 2000; Sheehan, 2001; Wagstaff and Goa, 2001). The utilization of depots has been variable and there has been a shift away from depots towards newer secondgeneration 'atypical' oral antipsychotics (Davis et al, 1994; Patel et al, 2003). This could be due to pain at injection site, as well as lack of reversibility and limited availability of antipsychotics in depot form (Bloch et al, 2001).

To address the need for further treatment options, a new method that is currently being developed involves a surgically implantable long-term medication delivery system (Siegel et al, 2002). This system would deliver psychiatric medication for up to 14 months after an implant has been inserted under the skin (Siegel et al, 2002; Kahn et al, in review). The intervention would involve a brief ambulatory surgical procedure with a local anesthetic, in which a small biodegradable implant would be inserted under the skin. In addition to assisting with medication adherence, this long-term delivery system would allow individuals to make treatment decisions during periods of health, rather than following symptom exacerbation (Siegel, 2002). It would also offer advantages of lower dosing and steady-state serum drug levels with fewer resultant side effects. Since implants could be used alone, or in conjunction with oral supplementation, it would also allow for dynamic dose adjustment. Furthermore, an implant would be advantageous over currently available depot formulations due to its reversibility, since the implant could be removed by a simple outpatient procedure. Implantable delivery systems are also more flexible than traditional decanoate depot formulations since they are able to incorporate a wider variety of medications without the constraints of forming ester bonds. Thus, a surgical implant could provide patients with a new method to receive uninterrupted access to medication for a year or more.

Although implants may be efficacious, they would only be effective if patients are willing to take them. Therefore, prior to offering the implant to humans, we aimed to assess patients' receptiveness towards this new method of medication administration. To meet this objective, our study involved designing and administering a survey that assessed patients' attitudes towards illness, medication, and the new surgically implantable medication delivery system. In order to understand the factors that contribute to a patient's decision about long-term medication implants, our survey included assessments of demographics, patient insight and attitude towards their illness, level of current and past medication adherence, attitudes towards psychiatric medicine, attitudes towards medicine in general, an understanding of the surgical implantation procedure, and attitudes towards the new surgical implantation intervention. Since self-report measures of adherence have been found to be as reliable as plasma measurement and electronic event monitoring techniques, we relied on patient self-report for medication adherence rates (Stephenson et al, 1993).

We hypothesized that a subgroup of patients with psychiatric illnesses would welcome this new method of receiving medications since it aims to aid in medication adherence and therefore improve outcome. Specifically, we hypothesized that past nonadherence would be positively correlated to favorably considering an implant. Secondly, we hypothesized that the diagnosis of a patient would influence the decision to consider an implant. We predicted that patients with life-long, chronic psychiatric illnesses such as schizophrenia, schizoaffective disorder, and bipolar disorder would be more likely to consider an implant $v s$ patients with more episodic illnesses, such as depression, anxiety, and those with other diagnoses. This is based on the assumption that people with illnesses that necessitate medication continuously would be more likely to consider a surgically implantable system than those who are not prescribed medication 'for life'. Thirdly, we hypothesized that insight would be positively correlated with patient decision to consider an implant. Fourthly, we hypothesized that severity of illness would be positively correlated with acceptance of an implant. We also analyzed several issues without directional hypotheses but for which this survey would generate hypotheses for future, large-scale, multinational studies. These include gender, age, past medication history, education, and race. Based on the outcome, we hope to generate a list of beliefs that could predict a patient's preference for favorably considering an implant.

\section{METHODS}

\section{Survey Development}

An Ovid Medline search was conducted of English language published articles from 1966 to 2001 using the following search words: schizophrenia (exp), questionnaires (exp), medication, and survey. Following this, a multiple choice and true/false based survey was developed based on guidance from the research literature coupled with clinical experience of the authors as described below. Fox's recommendations for high-quality questionnaire development were incorporated into the survey development process (Fox, 1996). These included neutral choices (such as 'don't know' and 'unsure'), mixed-response formats (open-ended and forced choice), clearly stated directions, use of general questions preceding more specific ones, 
numbering of questions, and the use of dark and highquality printing throughout the survey. We also balanced positively and negatively worded questions throughout the survey to assess the impact of wording and internal consistency. The survey was written at a 12 th grade reading level based on the SMOG readability formula (McLaughlin, 1969).

The primary outcome was measured by a question inquiring whether the patient would consider receiving their psychiatric medications through an implant. Since there is evidence that answers to earlier questions can affect responses to later questions, we created two versions of the survey (Foddy, 1993). In order to obtain an unbiased assessment of response on the primary outcome measure, the two questions immediately preceding the primary outcome measure were switched in Version A and B of the survey. These two questions inquired about reasons why patients might consider an implant and reasons why they might not. Response choices were based on expected arguments for and against implantable medicine, and also provided opportunities to indicate 'other' reasons.

The survey began with the collection of demographic information including gender, age, ethnicity, education, duration of illness, current psychiatric medications, and past psychiatric medications. Patient's self-reported diagnosis was also collected from a list of psychiatric illnesses, along with a question addressing patient's level of insight into their illness. The question 'I have a psychiatric illness' as well as a question identified as insight negative by Buchanan (1992), namely 'I do not have an illness for which I need psychiatric care', were both included to assess internal consistency. Insight into severity of illness was assessed with a question asking, 'How ill are you right now?'

Level of past medication adherence was assessed with a question asking, 'Since you first got ill, how careful have you been about taking your medicine?' Two of the response choices for this question were adapted from the Medication Adherence Questionnaire (MAQ) such that consistency in first-person format could be maintained throughout the survey (Morisky et al, 1986). The original MAQ \#2 'Are you careless at times about taking your medicine?' was changed to 'I have been careless at times about taking my medicine'. Original MAQ \#3 'When you feel better do you sometimes stop taking your medicine?' was adapted to 'When I felt better, I sometimes stopped taking my medicine'. Other choices included 'I have never missed my medicine' and 'I have taken my medicine for my psychiatric illness only when I felt bad'.

The level of current (defined as the proceeding 2 months) and past (defined as ever) medication compliance was assessed through a scaled question asking '... how often did you miss a dose of medicine?' with response choices of 0,25 , 50,75 , or $100 \%$. In order to understand reasons for medication nonadherence, a question was included that asked, 'When I miss my medicine the reasons are'. Two responses for this question were adapted from previous instruments, including MAQ item 'Do you ever forget to take your medicine?', which was changed to 'I forget to take my medicine', and DAI item 'I don't need medication once I feel better', which was changed to 'I do not need to take my medicine if I feel well' (Hogan et al, 1983). Additional choices included 'I prefer how I feel without my medicine', 'other people don't want me to take my medicine', 'voices tell me not to take my medicine', and 'I never miss my medicine'.

The next group of questions were designed to assess attitudes towards psychiatric medicine in order to determine the effect of these attitudes on acceptance of long-term delivery systems. A question adapted from Mantonakis et al (1985) assessed whether the patient believed that continuous use of medicine was necessary. Furthermore, Hogan et al (1983)'s 'subjective positive' item 'good things about medication outweigh the bad' was also included as a True/ False option. Additional questions that were included based on clinical experience, assessed whether the patient thought it was necessary to take medicine for the treatment of his/ her psychiatric illness and whether the patient believed that he/she will get sick if he/she stopped taking his/her medicine. Attitudes towards medicine in general were also included to assess whether attitudes towards psychiatric medications differed from beliefs about medication in general. Questions included 'I prefer not to take medicine for any medical problems (for example, an infection, pain, high blood pressure, diabetes, etc) and 'when I am ill, I usually take medicine to make me better (for example, when I have an infection, pain, high blood pressure, diabetes, etc)'

We also included several questions that were repeated in various forms to assess internal consistency of responses. For example, DAI item 'I do not need my medicine once I feel better' was repeated as a True/False option in a later question. Additionally, in another question, it was converted to positive phrasing. The option 'I never miss my medicine' was included in three of the adherence questions with multiple choices to assess consistency of response. Two questions that assessed attitudes towards medicine in general were phrased in the positive and negative to assess consistency.

Questions were included to assess whether or not patients had a good understanding of the surgical implantation procedure, prior to asking them to make a decision about accepting an implant. A brief description about the procedure was provided in outline format as follows.

- We are creating a way to give medicine for many months.

- This method uses a small biodegradable tablet that is placed under the skin (implant).

- This procedure would provide medicine for many months after a brief surgical procedure.

- The surgery would take about $15 \mathrm{~min}$ with a local anesthetic (the patient will not go to sleep).

- The implant can be placed almost anywhere on the body, such as the inner arm, the thigh, etc.

- Risks of the surgery could include minor infections of the skin, irritation of the skin, a small scar (approximately $1 / 2$ inch) and an adverse reaction to the local anesthetic.

- If necessary, the implant can be removed by a physician.

Following that, five questions were extracted directly from the description in order to assess the patient's level of understanding of the surgical implantation procedure (Table 1). The questions were all stated in the positive 
Table I Survey Questions and Responses

\begin{tabular}{|c|c|c|}
\hline Insight questions & $\%$ & Consider in \\
\hline \multicolumn{3}{|l|}{ I have a psychiatric illness } \\
\hline Yes & 92.6 & 46.2 \\
\hline No & 7.4 & 20.0 \\
\hline \multicolumn{3}{|l|}{ I have } \\
\hline Schizophrenia & 44.8 & 39.0 \\
\hline Schizoaffective disorder & 13.5 & 56.5 \\
\hline Bipolar disorder & 13.0 & 48.0 \\
\hline Depression & 21.4 & 48.7 \\
\hline Anxiety disorder & 2.1 & 25.0 \\
\hline Other/none & 5.2 & 27.8 \\
\hline \multicolumn{3}{|c|}{ I do not have an illness for which I need psychiatric care } \\
\hline Yes & 14.5 & 36.0 \\
\hline No & 85.5 & 44.1 \\
\hline \multicolumn{3}{|l|}{ How ill are you right now? } \\
\hline I am very ill right now & 8.9 & 56.3 \\
\hline I am moderately ill right now & 17.3 & 38.2 \\
\hline I am somewhat ill right now & 15.8 & 58.6 \\
\hline I am mildly ill right now & 33.2 & 40.6 \\
\hline I am not ill right now & 24.8 & 34.8 \\
\hline
\end{tabular}

Medication adherence questions

$\%$

Since you first got ill, how careful have you been about taking your medicine?

I never miss my medicine

I have taken my medicine for my psychiatric illness only when I felt bad

I have been careless at times about taking my medicine*

When I felt better, I sometimes stopped taking my medicine

Over the past 2 months, how often did you miss a dose of medicine?*****

$0 \%$ (I never miss a dose of medicine)

$25 \%$ (I sometimes miss a dose of medicine)

$50 \%$ (I miss taking my medicine half of the time

75\% (I miss a dose of medicine most of the time)

100\% (I always miss taking my medicine)

When I miss my medicine the reasons are

I never miss my medicine*

The medicine makes me feel bad (side effects)

I do not need to take my medicine if I feel well

I forget to take my medicine ${ }^{* *}$

I run out of my medicine***

I prefer how I feel without medicine

Other people do not want me to take my medicine 
Table I (Continued)

\begin{tabular}{|c|c|c|}
\hline Attitudes towards medication & $\%$ True & Consider implant \\
\hline It is necessary to take medicine for the treatment of my psychiatric illness & 83.3 & 46.3 \\
\hline $\begin{array}{l}\text { I prefer not to take medicine for any medical problems (for example, an } \\
\text { infection, pain, high blood pressure, diabetes, etc) }\end{array}$ & 18.3 & 34.4 \\
\hline $\begin{array}{l}\text { When I am ill, I usually take medicine to make me better (for example, } \\
\text { when I have an infection, pain, high blood pressure, diabetes, etc) }\end{array}$ & 88.1 & 45.2 \\
\hline Comprehension questions & $\%$ Correct & Consider implant \\
\hline A surgical implant would be placed under the skin* & 86.8 & 47.2 \\
\hline $\begin{array}{l}\text { Inserting the implants would require a 15-min surgery with a local } \\
\text { anesthetic }\end{array}$ & 84.6 & 46.2 \\
\hline The implant is removable if necessary by a physician & 92.2 & 46.2 \\
\hline The medicine is delivered for many months & 86.5 & 44.7 \\
\hline $\begin{array}{l}\text { The risks of the surgical procedure include minor infections of the skin, } \\
\text { irritation at site, and a small scar }\end{array}$ & 88.3 & 44.6 \\
\hline
\end{tabular}

Reasons I would consider receiving medicine for my psychiatric illness from an implant

$\begin{array}{lr}\text { I do not like what happens when I miss my medicine*** } & 43.9 \\ \text { I would prefer not to take medicine everyday by mouth** } & 58.3 \\ \text { I have difficulty taking my medicine every** } & 76.0 \\ \text { To help me stay well** } & 69.0 \\ \text { There would be less burden on my family if I got an implant*** } & 57.9 \\ \text { Other } & 62.6 \\ \end{array}$

Reasons I would not consider receiving medicine for my psychiatric illness from an implant

I do not want to try something new**

$30.4 \quad 16.4$

I prefer taking my medicine by mouth***

I do not want to feel controlled*

I would never have surgery for anything, even if my life depended on it

I am concerned about the side effects of surgery

* Indicates a correlation between the patient's answer to the question and a decision to accept implant $(p<0.05)$. ${ }^{[2]} * * *$ indicates a correlation between the patient's answer to the question and a decision to accept implant $(p<0.01)$. ${ }^{[3]} * * *$ Individual variables do not correlate with a decision to accept implant. However, analysis of 'never missing meds' and 'missing meds one or more times' reveals a correlation between never having missed medicine and accepting the implant ( $p=0.017)$.

(ie all True responses) so that misleading information about the surgical implant procedure was not suggested.

\section{Survey Administration}

All procedures and surveys were approved by the Office of Regulatory Affairs at the University of Pennsylvania to comply with Federal guidelines for research involving human subjects. Individuals with schizophrenia, schizoaffective, mood and anxiety related psychiatric disorders were invited to complete the brief paper and pencil version of the 'Attitude Survey for Psychiatric Medicine Implant'. Participants were randomly provided with Version A or Version B of the survey, where the order of the questions, inquiring about reasons for and against an implant, were switched. There were five venues for approaching patients to complete the survey. These included an outpatient clinic within a Schizophrenia Research Center, a nonresearchoriented general outpatient clinic, in-patient hospital units at a private community hospital and a tertiary care medical center, a community based mental health facility, and community educational conferences. Patients were assessed for their ability to participate in a research survey by their treating physician prior to being approached at all sites, except for the community educational conferences where surveys were distributed through handouts. Either a research coordinator or the patient's doctor administered the surveys. This was done to ensure that patients were clinically stable and able to participate in research. This assessment also served to ensure that no patient had the 
misperception that they were being offered an implant at the current time. Patients were presented with the choice of completing the survey anonymously or through nonanonymous consenting procedures. In either case, the patient was assured that his/her treating psychiatrist would not have access to his/her survey. This was done to maintain confidentiality and to enable the patient to provide unbiased answers. For a subset of patients, a corresponding physician verification form was also administered to obtain assessments from the treating psychiatrist regarding diagnosis.

\section{Data Analysis}

A two-sample $t$-test was used to compare the mean age between patients who would consider an implant $v s$ patients who would not consider an implant. Fisher's exact tests were used for assessing the association between categorical variables. For assessing internal consistency in the survey, Kappa statistics were calculated to measure the strength of agreement between responses to similar questions in which patients should have answered identically. Additionally, McNemar's test was used for comparing the proportion of patients missing current $v s$ past medication use. Analyses were conducted using SAS version 8.1 (SAS Institute, Cary, $\mathrm{NC})$.

\section{RESULTS}

A total of 206 surveys were completed. As indicated in Table 1, the sample consisted mainly of patients who reported diagnoses of schizophrenia or schizoaffective disorder, and also included patients with bipolar disorder, depression, and anxiety. The mean age of the respondents was $40 \pm 12$ with $50.7 \%$ male and $49.3 \%$ female respondents. The respondents included 53.9\% Caucasians, $40.7 \%$ African Americans, 2.5\% Asians, and 2.9\% 'Other'. Additionally, the highest proportion of patients reported completing more than high school $(45.1 \%)$, followed by those who reported completing high school (35.9\%), middle school (15.9\%), and less than eighth grade $(3.1 \%)$. The majority of the patients reported a duration of illness greater than 10 years $(59.6 \%)$, followed by 3-5 years (16.1\%), 6-10 years (12.4\%), 1-2 years $(7.8 \%)$, and less than 1 year $(4.2 \%)$. Locations where the surveys were collected included a community mental health center $(34.5 \%)$, research setting $(23.3 \%)$, nonresearch outpatient clinic (18.5\%), in-patient $(13.6 \%)$, and an educational conference (10.2\%). The relationship between each item and the patient's decision to consider an implant was analyzed. No demographic item was significantly correlated with the final decision to consider an implant.

Patients were then asked to report their current and past medications. Most patients reported taking multiple current $(60.2 \%)$ and past $(72.8 \%)$ medications, and there was inadequate power to analyze each individual medication's effect on a person's decision to consider an implant. We grouped the medications as follows: older antipsychotics (13.6\% current, $54 \%$ past), Clozaril (13.6\% current, $15.8 \%$ past), newer antipsychotics (60.7\% current, $66.3 \%$ past), mood stabilizers (31.9\% current, $48.5 \%$ past), antidepressants $(39.8 \%$ current, $58.9 \%$ past $)$, anxiolytics (1.1\% current, $16 \%$ past), other ( $24.6 \%$ current, $24.2 \%$ past), and unsure (1.6\% current, $2.4 \%$ past). There was no significant relationship between a history of taking any specific class of agent and current attitudes towards implantable delivery systems. The only significant relationship was for patients who reported taking 'other' medications in the past. Of note, $92.7 \%$ of patients were able to identify their current medications, while only $80.1 \%$ were able to identify past medications (McNemar's test: $S=18.8,1 \mathrm{df}, p<0.01$ ).

Table 1 reports the survey questions along with the response rate and relationship to implant decision for each question. On questions inquiring about insight about their psychiatric illness, $92.6 \%$ of patients endorsed that they had a psychiatric illness when this question was asked in a positive phrasing, while $85.5 \%$ acknowledged being ill in response to a negatively phrased question. Of the individuals who reported that they did not have a psychiatric illness in the positively worded question, $66.7 \%$ were in the none/missing/other category for self-assigned diagnosis. Only 1.5 and $5.3 \%$ of patients with schizophrenia and bipolar disorder, respectively, were likely to state that they did not have a psychiatric illness, and none of the schizoaffective patients denied having a psychiatric illness. In contrast $16.7 \%$ of patients with depression denied having a psychiatric illness. However, when asked whether they have an illness for which they need psychiatric care, $17.1 \%$ of patients with schizophrenia and $12.0 \%$ with schizoaffective disorder denied having an illness and needing care. Additionally, $4.0 \%$ of bipolar patients and $10.3 \%$ of depressed patients also reported that they did not have an illness that needed psychiatric care. However, neither a single diagnosis $(p=0.399)$ nor insight $(p=0.186)$ was predictive of one's decision regarding considering an implant. Data also indicate that individuals who endorsed having a psychiatric illness were significantly more likely to report that the continuous use of medication is necessary for the treatment of their illness $(p=0.004)$, and that it is necessary to take medication for their psychiatric illness $(p=0.005)$. These individuals also report that they usually take medicine for nonpsychiatric conditions when they are medically ill $(p<0.001)$. Table 1 also lists the results of patients' self-assessment of illness severity. Although the majority of patients identified themselves as being mildly ill or not ill (57.9\%), there was no relationship between severity of illness and decision regarding implants $(p=0.222)$.

Table 1 also reports patients' level of medication adherence, reasons for missing medication and the relationship of these variables to implant decision. The majority of patients reported that they have never missed their medications (56.8\%) since they first got ill. A larger proportion $(61.7 \%)$ also reported that they had not missed their medication during the last 2 months. Many patients reported being careless at times $(29.2 \%)$ and stopping their medication when they felt better $(17.6 \%)$. Current medication adherence was not correlated with patients' decision regarding implantable delivery systems (Fisher's exact, $p=0.065)$. However, after grouping those who reported missing medications and those who reported not missing medications, there was a significant relationship between medication adherence and attitudes towards implants (Fisher's exact, $p=0.017$ ). Patients who reported never 
missing any medications were less likely to consider implantable medicine. In patients with anxiety and depression, 100 and $65 \%$ were likely to report that they never missed their medicine, followed by none/missing/other (60\%), schizophrenia (58.8\%), and schizoaffective disorder (42.3\%). Patients with bipolar disorder (41.7\%) were least likely to report that they never missed their medicine. Patients who reported that they forget to take their medications and run out of medications were significantly more likely to accept implantable medicine $(p=0.004$ and 0.005). Patients with schizophrenia/schizoaffective/bipolar disorder were more likely to state that they missed their medication due to forgetting $(35.8 \%)$ or running out (20.2\%), than patients with depression/anxiety/none/other/ missing (23.1\% - forgetting, 15.4\% - running out). Interestingly, neither psychotic symptoms nor perceived external influences were frequently reported as reasons for missing medication.

Patient attitudes towards both psychiatric and nonpsychiatric illnesses are also listed in Table 1. While a large proportion of participants who responded to this question reported the need for continuous use of medication $(90.1 \%)$, an equally large group also reported that they did not need psychiatric medication once they felt better $(89.2 \%)$ and that they would not get sick if they stopped taking psychiatric medicine $(82.7 \%)$. Although patients with schizophrenia were most likely to endorse this trend, there was no significant effect of diagnosis on this relationship. Most patients reported that they prefer to take medicine for nonpsychiatric conditions (81.7\%). On questions related to attitudes towards medication, only patients who reported that the good things about their psychiatric medication outweighed the bad were significantly more likely to consider implantable medicine $(p=0.007)$.

The next series of questions were designed to assess the level of comprehension regarding the implant procedure. Table 1 shows the percentage of patients who correctly answered each question by choosing 'True'. There was an average accuracy of $87.7 \%$ when answering questions about the implants. Data were also analyzed to assess the proportion of patients who correctly answered $0,1,2,3,4$, or 5 questions correctly. Overall, $86.2 \%$ of the patients answered four or more questions correctly, while 4.2, 3.2, $1.6,4.8 \%$ respectively answered $3,2,1,0$ questions correctly. Patients with schizophrenia and schizoaffective disorder got all five correct 76.0 and $73.9 \%$ of the time, respectively, while patients with depression got all five correct $82.1 \%$ of the time. The none/missing/other group accounted for most of the errors, where they got all five correct only $55 \%$ of the time. When a grouped analysis was conducted, there was a significant difference $(p=0.002)$ between patients with schizophrenia, schizoaffective, and bipolar disorders, vs patients with depression, anxiety, and none/ other/missing. The depression/anxiety/none/other/missing group was more likely to get all answers wrong as compared to the schizophrenia/schizoaffective/bipolar subgroup. The none/other/missing group accounted for most of these errors. When a further exploratory analysis was conducted, we found that of the 24 individuals in the none/other/ missing group, 13 were diagnosed as having schizophrenia or schizoaffective disorder by their physician. There were only three patients out of this group whose self-reported diagnosis matched the diagnosis of their physician, while for eight out of the 24 individuals there was no physician verification form completed. Additionally, we found that one specific answer in the implant comprehension questions was associated with a significant difference between both the diagnostic subgroups and attitudes towards implants. An incorrect 'false' response to the question inquiring whether a surgical implant would be placed under the skin was correlated with patient's decision not to accept an implant $(p=0.016)$. There was also a nonsignificant trend for patients not to consider an implant if they did not understand that the implant is removable by a physician $(p=0.059)$. The depression/anxiety/none/ missing/other subgroup was more likely to get this answer wrong than the schizophrenia/schizoaffective/bipolar subgroup $(p=0.040)$. Patients who identified themselves as having a psychiatric illness were significantly more likely to answer correctly that the implant is removable by a physician $(p=0.013)$.

Furthermore, a large proportion of patients claimed that the main reasons they would favorably consider an implant were to help them stay well and to avoid the adverse consequences of missing their medications. Additionally, many patients attribute reluctance to consider implants to a resistance to try something new, a preference for taking medication by mouth, and concern about the side effects of surgery. Resistance to try something new was most pronounced for patients with schizophrenia/schizoaffective/bipolar $(p<0.01)$. Additionally, patients in the schizophrenia/schizoaffective/bipolar group were also more likely to report not wanting to feel controlled as a reason for not accepting an implant $(p=0.027)$. Among identified reasons for and against implants, only concern about surgery and its side effects was unrelated to the decision. Additionally, among those favorable towards an implant, 'other' reasons were also unrelated to the decision.

The final question and the primary outcome measure asked patients if they would consider receiving medicine for their psychiatric illness from a surgical implant. These data indicate that $42.9 \%$ of patients reported that they would favorably consider it, while $57.1 \%$ of patients reported that they would not.

In order to assess internal consistency, the answer 'I never miss my medication' was repeated throughout the survey. We found that only $2.5 \%$ of patients were not internally consistent on this measure. When the same question was repeated later on two occasions, patients consistently reported that they never missed their medicine $(\kappa=0.616$, $0.499,0.537)$. Similarly, there was internal consistency in responses between patients who reported that they prefer to take medicine for any medical problem and those who reported taking medicine when they are medically ill $(\kappa=0.426)$. These results indicate a moderate to substantial degree of internal agreement among patients, suggesting that the data obtained with this instrument are reliable (Landis and Koch, 1977). Our results also indicated that there is no significant effect for the version of survey administered. Specifically, there was no difference in outcome whether patients were asked reasons for or against an implant just prior to being asked whether they would consider an implant $(p=0.660)$. 


\section{DISCUSSION}

This survey examined patients' attitudes and beliefs towards illness, medication, and surgically implantable medication. We found that patients are almost equally split between favorably and not favorably considering an implant. There are distinct variables that differentiated the two groups of patients. We suggest that demographics, diagnosis, duration of illness, severity of illness, and type of health care setting do not influence patients' decisions regarding implantable medicine. Additionally, despite the difficulties in the ability to report on past (even defined as past 2 months) $v s$ current medications, a history of taking any specific class of agent did not influence views on the implant. This interpretation is complicated by the observation that the majority of patients reported taking several medications concomitantly. Since implants are capable of incorporating multiple medications in a single formulation, this offers an additional benefit to assist patients with complicated dosing regimens. We also suggest that medication nonadherence and a subjective recognition of the benefits of medication are positively correlated with the decision regarding implants. Patients who ascribe reasons such as forgetting to take medicine or failing to obtain refills in time are also most favorable towards implants.

Overall, there were high rates of accuracy on questions assessing comprehension of the implant procedure and delivery system. Understanding that a surgical implant would be placed under the skin was key to a positive correlation with accepting an implant. The decision to consider an implant was related to a patient's desire to avoid the adverse consequences of missing medicine, stay well, avoid the need to take daily medications by mouth, decrease the burden on his/her family, and get help with the difficulty of taking medicine everyday. The decision not to consider an implant was related to a preference to take medicine by mouth, a concern about feeling controlled, other subjective reasons, and a desire not to try something new. Neither the concept of surgery nor its side effects were correlated with patients' decision to consider implantable medicine.

In addition to investigating attitudes towards implants, we also found that patients with chronic psychiatric illnesses (schizophrenia/schizoaffective/bipolar disorder) were more likely to have a lack of insight into the need for psychiatric care, rather than a lack of insight into having a psychiatric illness. In contrast, individuals with depression were less likely to affirm that they have an illness and need psychiatric care, perhaps due to a preference for viewing their condition as being state dependent rather than a 'psychiatric illness'. Medication adherence was also influenced by chronicity of diagnosis, with greater nonadherence in the bipolar/schizoaffective/schizophrenia subgroup than the anxiety/depression group.

Although the role of side effects in medication adherence is unclear (Hogan et al, 1983; Buchanan, 1992; Awad, 1993; Cabeza et al, 2000), our results suggest that neither side effects nor psychotic symptoms play a major role in the decision regarding implants in this cohort of patients. However, the current survey did not ask patients to report on which specific side effects they experienced. As such, we are unable to address the role of specific types of side effects on either medication adherence or implant decision in the current report. Similarly, we did not assess the duration of previous medication exposure and cannot comment on the effect of duration of medication on any outcome measure. However, future studies could incorporate questions regarding both duration of medication exposure and specific medication side effects to assess the effects of each of these variables on implant decision. We also found that although patients may lack insight into the consequences of nonadherence with psychiatric medicine, this does not necessarily extend to nonpsychiatric conditions.

As such, along with an exploration of illness and medication adherence, this survey outlines the characteristics of a subgroup of patients who are willing to consider implantable medicine. We have highlighted several variables that influence patients' decision to consider a surgically implantable medication delivery system. While this study was limited to a sample of relatively well patients from an urban northeastern area in the United States, future studies incorporating a larger, internationally representative sample will allow the investigation of additional variables not addressed here. For example future studies could investigate the role of individual medications and depot formulations as well as illness severity on the decision to consider an implant. Such studies could also incorporate methods designed to verify patient's reports on diagnosis, severity, and adherence. Furthermore, the role of side effects in implant decisions could also be explored further. Finally, the attitudes and beliefs of family members and health care providers towards this new treatment method are also important to investigate, since these individuals would be affected by this new delivery method. As such, these results provide the foundation for future work that can further elucidate attitudes and beliefs towards illness, medication, and this new treatment method.

\section{ACKNOWLEDGEMENTS}

All funding for this work was provided by a Center Grant from The Stanley Medical Research Institute (SJS).

\section{REFERENCES}

Adams J, Scott J (2000). Predicting medication adherence in severe mental disorders. Acta Psychiatr Scand 101: 119-124.

Adams Jr SG, Howe JT (1993). Predicting medication compliance in a psychotic population. J Nerv Ment Dis 181: $558-560$.

Archer DF, Bigrigg A, Smallwood GH, Shangold GA, Creasy GW, Fisher AC (2002). Assessment of compliance with a weekly contraceptive patch (Ortho Evra/Evra) among North American women. Fertil Steril 77: S27-S31.

Awad AG (1993). Subjective response to neuroleptics in schizophrenia. Schizophr Bull 19: 609-618.

Becker MH, Maiman LA (1975). Sociobehavioural determinants of compliance with health and medical care reccommendations. Med Care 13: 10-24.

Bergen J, Hunt G, Armitage P, Bashir M (1998). Six-month outcome following a relapse of schizophrenia. Aust $N Z J$ Psychiatry 32: 815-822.

Bloch Y, Mendlovic S, Strupinsky S, Altshuler A, Fennig S, Ratzoni $G$ (2001). Injections of depot antipsychotic medications in patients suffering from schizophrenia: do they hurt? J Clin Psychiatry 62: 855-859. 
Buchanan A (1992). A two-year prospective study of treatment compliance in patients with schizophrenia. Psychol Med 22: 787-797.

Budd K (2003). Buprenorphine and the transdermal system: the ideal match in pain management. Int J Clin Pract 133: 9-14.

Cabeza IG, Amador MS, Lopez CA, Gonzalez de Chavez M (2000). Subjective response to antipsychotics in schizophrenic patients: clinical implications and related factors. Schizophr Res 41 : 349-355.

Casper ES, Regan JR (1993). Reasons for admission among six profile subgroups of recidivists of inpatient services. Can $J$ Psychiatry 38: 657-661.

Cramer JR, Rosenheck R (1998). Compliance with medication regimens for mental and physical disorders. Psychiatric Serv 49: 196-201.

David AB, Buchanan A, Reed A, Almeida O (1992). The assessment of insight in psychosis. Br J Psychiatry 161: 599-602.

David AS (1990). Insight and psychosis. Br J Psychiatry 156: 798-808.

Davis JM, Matalon L, Watanabe MD, Blake L, Metalon L (1994). Depot antipsychotic drugs. Place in therapy. Drugs 47: 741-773.

Fant RV, Owen LL, Henningfield JE (1999). Nicotine replacement therapy. Primary Care 26: 633-652.

Foddy W (1993). Constructing Questions for Interviews and Questionnaires: Theory and Practice in Social Research. Cambridge University Press: Cambridge, UK.

Fox C (1996). Questionnaire development. J Health Soc Policy 8: $39-48$.

Francis VK, Korsch BM, Morris MJ (1969). Gaps in doctor-patient communications: patients' response to medical advice. $N$ Engl $\mathrm{J} \mathrm{Med}$.

Ghaemi SPHJ (1994). Lack of insight in psychotic and affective disorders: a review of empirical studies. Harvard Rev Psychiatry 2: 22-33.

Gupta RK, Chang AC, Siber GR (1998). Biodegradable polymer microspheres as vaccine adjuvants and delivery systems. Dev Biol Stand 92: 63-78.

Hackett D (2000). Venlafaxine XR in the treatment of anxiety. Acta Psychiatr Scand 406(Suppl): 30-35.

Hogan TP, Awad AG, Eastwood R (1983). A self-report scale predictive of drug compliance in schizophrenics: reliability and discriminative validity. Psychol Med 13: 177-183.

Kahn JB, Winey KI, Liang Y, Maxwell CR, Weightman BD, Pollock $\mathrm{BG}$ et al. In vivo demonstration of surgically-implantable, longterm antipsychotic delivery systems. (in review).
Landis JR, Koch GG (1977). The measurement of observer agreement for categorical data. Biometrics 33: 159-174.

Mantonakis JM, Markidis M, Kontaxakis V, Liakos A (1985). A scale for the detection of negative attitudes towards medication among relatives of schizophrenic patients. Acta Psychiat Scand 71: 186-189.

McLaughlin G (1969). SMOG grading: a new readability formula. J Reading 12: 639-646.

Morisky DEG, Lawrence W, Levine DM (1986). Concurrent and predictive validity of a self-reported measure of medication adherence. Med Care 24: 67-74.

NIMH (2000). Publication PA-00-016.

Patel MX, Nikolaou V, David AS (2003). Psychiatrists' attitudes to maintenance medication for patients with schizophrenia. Psychol Med 33: 83-89.

Sheehan DV (2001). Attaining remission in generalized anxiety disorder: venlafaxine extended release comparative data. J Clin Psychiatry 62(Suppl 19): 26-31.

Siegel SJ (2002). Increased patient automony through long-term antipsychotic delivery systems in schizophrenia. Expert Rev Neurotherap 2: 89-91.

Siegel SJ, Winey KI, Gur RE, Lenox RH, Bilker WB, Ikeda D et al (2002). Surgically implantable long-term antipsychotic delivery systems for the treatment of schizophrenia. Neuropsychopharmacology 26: 817-823.

Smith CM, Barzman D, Pristach CA (1997). Effect of patient and family insight on compliance of schizophrenic patients. J Clin Pharmacol 37: 147-154.

Stephenson BJ, Rowe BH, Haynes RB, Macharia WM, Leon G (1993). The rational clinical examination. Is this patient taking the treatment as prescribed? JAMA 269: 2779-2781.

Valenstein M, Copeland LA, Owen R, Blow FC, Visnic S (2001). Adherence assessments and the use of depot antipsychotics in patients with schizophrenia. J Clin Psychiatry 62: 545-551.

Viguera AC, Baldessarini RJ, Hegarty JD, van Kammen DP, Tohen M (1997). Clinical risk following abrupt and gradual withdrawal of maintenance neuroleptic treatment. Arch Gen Psychiatry 54: 49-55.

Wagstaff AJ, Goa KL (2001). Once-weekly fluoxetine. Drugs 61: 2221-2228; discussion 2229-2230.

Wei FC, Jann MW, Lin HN, Piao-Chien C, Chang WH (1996). A practical loading dose method for converting schizophrenic patients from oral to depot haloperidol therapy. J Clin Psychiatry 57: 298-302.

Weiden PJ, Olfson M (1995). Cost of relapse in schizophrenia. Schizophr Bull 21: 419-429. 